\title{
Postoperative Functional and Radiological Outcome Comparison of Computer-assisted and Conventional Total Knee Arthroplasty at 6 Months Follow-up: A Cross Sectional Study
}

\author{
Budimansyah $\mathrm{M}^{1}$, Ismail Hadisoebroto Dilogo ${ }^{2}$, Sumargono $\mathrm{E}^{3}$ \\ ${ }^{1}$ Orthopaedic division, Department of Surgery, Persahabatan General Hospital, Jakarta, Indonesia \\ ${ }^{2} \mathrm{Hip} \&$ Knee Adult reconstruction division, Department of Orthopaedic and Traumatology, Faculty of Medicine Universitas Indonesia, Cipto \\ Mangunkusumo General Hospital, Jakarta, Indonesia \\ ${ }^{3}$ Adult reconstruction division, Department of Orthopaedic and Traumatology, Saint Carolus Hospital, Jakarta, Indonesia
}

\begin{abstract}
Background:Computer-assisted navigation TKA has just been adapted in Indonesia for the last decade. The method is aiming to achieve more precise mechanical alignment in TKA. We conducted this study to compare the functional and radiological outcome of TKA using computer-assisted navigation with the conventional method.

Method: Fifty elderly patients aged more than 50 years old with severe knee osteoarthritis (Kellgren Lawrence stage IV) who scheduled for TKA surgery in the first half of 2019 were consecutively sampled. All surgeries were done with standard medial parapatellar approach using Press-fit condylar (PFC) system total knee arthroplasty (Johnson \& Johnson Professional, Raynham, MA). Brainlab Knee3 (version 3.2) navigation software was used for computer-assisted navigation group. All patients were measured at 6 months post-operative follow up for objective functional status with Western Ontario and McMaster Universities Index (WOMAC) and Oxford Knee Score (OKS). Weight-bearing, long-leg follow-up radiographs of both lower limbs were taken, and coronal tibiofemoral angle (CTFA), coronal femoral component angle (CFCA) and coronal tibial component angle (CTCA) were measured.

Results: Group of computer-assisted navigation TKA surgery was found to have significantly lower WOMAC score $(p<0.05)$ and higher OKS score $(p<0.05)$. Although not statistically significant, greater angle of deviation is observed in the conventional TKA surgery group. Proportions with deviation of less than 3 o were found more in the group using computer navigation.

Conclusion. Patients who had TKA surgery under computer-assisted navigation would benefit a better functional outcome if compared with conventional TKA surgery, as measured with WOMAC and OKS. However, there is no significant difference in terms of radiographic value of coronal-plane mechanical deviation on both methods.
\end{abstract}

Keywords: osteoarthritis, computer-assisted navigation surgery, total knee replacement, total knee arthroplasty, functional outcome, knee mechanical axis

Level of Evidence: III

This is an open access article under the CC-BY-SA license.

\section{Article history}

Submitted: July $1^{\text {st }}, 2020$

Revise : July $20^{\text {th }}, 2020$

Accepted. : July $21^{\text {st }}, 2020$

\section{Corresponding Author:}

Muhammad Budimansyah, MD. Jl. Persahabatan Raya No. 1, Rawamangun, DKI Jakarta 13230; Phone: (62-21) 4891708 ext. 146 Email: adinbudi_8910@yahoo.com 


\section{Introduction}

Total knee arthroplasty (TKA) is one of the most successful orthopaedic surgical intervention in history, with $90 \%$ successful rate in 10 years. ${ }^{1,2}$ This rate of success depends on the surgical technique, soft tissue condition, perioperative care and the patient itself. ${ }^{1}$ Failure to achieve a good mechanical alignment in TKA may lead to excessive wear, early component loosening and patella-femoral disturbance. Mechanical alignment of more than $3^{\circ}$ was found to decrease the 10 -year successful rate up to $75 \% .^{3}$ This alignment can be evaluated extramedullary and intramedullary. However, this evaluation depends on the surgeon capability and knee joint anatomy, which differs on each patient and may be altered along with osteoarthritis progression.

In the last two decades, the world has been introduced with computer-assisted navigation method, aiming to achieve more precise mechanical alignment in TKA. The navigation system is able to assess and display real time information of the bony structure, including alignment and rotation. Therefore, it is able to increase the accuracy of prosthetic selection and placement, which correlates with good functional outcome. ${ }^{4,5}$ However, controversies still exist up to now. One meta-analysis found that computerassisted navigation has no difference in terms of radiological outcome, increase the operation time and also not cost-effective. ${ }^{6}$

Computer-assisted navigation TKA has just been adapted in Indonesia for the last decade. Unlike years ago, there has been an increase in training as well as technical support, and many orthopedic surgeons have been familiarized with the system. Our study aims to re-evaluate the short term functional outcome of computerassisted and conventional TKA in Indonesian population.

\section{Methods}

This analytic cross-sectional study was conducted at Saint Carolus hospital, Jakarta. Fifty elderly patients aged more than 50 years old with severe knee osteoarthritis (Kellgren Lawrence stage IV) who scheduled for TKA surgery in the first half of 2019 were consecutively sampled. Surgeries were performed by single orthopaedic surgeon. All patients were allocated into groups of computer-assisted TKA and conventional TKA. According to sample size calculation, a minimum number of 20 patients on each group is required for the study. Patients with history of infection, bilateral surgery, history of previous knee surgery, or rheumatoid arthritis were excluded.

All surgeries were done with standard medial parapatellar approach. Press-fit condylar (PFC) system total knee arthroplasty (Johnson \& Johnson Professional, Raynham, MA) was used in both groups regardless of cruciate ligament retaining or sacrifice. Brainlab Knee3 (version 3.2) navigation software was used for computerassisted navigation group.

All patients were measured for objective functional status with Western Ontario and McMaster Universities Index (WOMAC) and Oxford Knee Score (OKS). WOMAC is a simple selfadministered questionnaire that is used to evaluate the function in hip and knee osteoarthritis patient. It is divided into 3 subscales of pain ( 5 items), stiffness ( 2 items) and physical function (17 items). Each items are scored on a scale of $0-4$, with the highest score is 96 . Lower overall score indicates better functional outcome. OKS is a patient-reported scoring system to assess function and pain after TKA surgery. It consists of 5 items for pain evaluation and 7 items for function evaluation. Each items are scored on a scale of $0-4$, with the highest score of 48 . Higher overall score indicates better functional outcome. ${ }^{7}$ 
Weight-bearing, long-leg follow-up radiographs of both lower limbs were taken, and coronal tibiofemoral angle (CTFA), coronal femoral component angle (CFCA) and coronal tibial component angle (CTCA) were measured. CTFA is defined as the angle that is formed by femoral and tibial mechanical axes as assessed on the lateral side of the midline. Femoral mechanical axis is acquired by line forming from the center head of femur to the center of knee joint. Tibia mechanical axis is acquired by line forming from center of knee joint to the center of ankle joint. Neutral value of CTFA is defined as $180^{\circ}$. CFCA is defined as the angle that is formed by femoral mechanical axis and the trans condylar line of the femoral component as assessed on the lateral side of the midline. Neutral value of CFCA is defined as $90^{\circ}$. CTCA is defined as the angle that is formed by tibial mechanical axis and the tibial base plate as assessed on the lateral side of the midline. Neutral value of CTCA is defined as $90^{\circ}$. Good mechanical alignment is considered when the deviation of all three angles are within $3^{\circ}$ from neutral. ${ }^{8}$
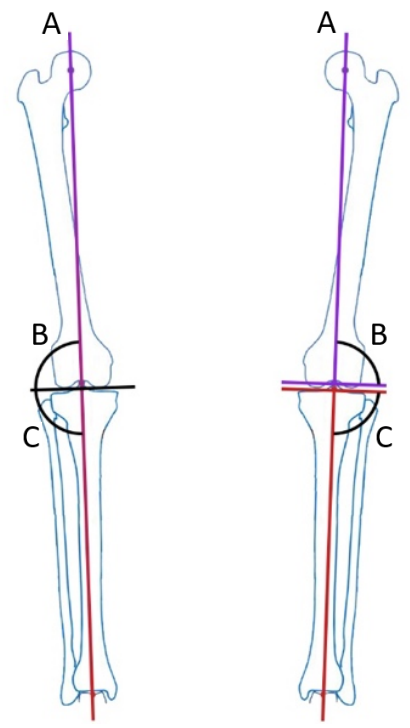

Figure 1. Weight-bearing, long-leg radiographs of lower limbs measurement: A. coronal tibiofemoral angle (CTFA); B. coronal femoral component angle (CFCA); and C. coronal tibial component angle (CTCA).
Data analysis was performed by using Statistical Program for Social Science $\left(\right.$ IBM $\left.^{\circledR}\right)$ v.20.00. Categorical data is presented as sample size (n) and percentage (\%). Distribution of numerical data was analyzed by using Saphiro-Wilk test. Normally distributed data is presented as mean and standard deviation value, while abnormally distributed data is presented as minimum and maximal value. Pearson Chi-Square test or Fisher exact test was used to analyze proportional difference between variables. Unpaired T-test was used to compare the means of two normally distributed groups. Mann-Whitney test was used to compare the means of two abnormally distributed groups. A p-value less than 0.05 (typically $\leq 0.05$ ) is considered as statistically significant.

\section{Results}

The characteristics of the subjects in our study are summarized in Table 1. Fifty patients who had TKA surgery in our hospital on the first half of 2019 were recruited to the study. Patients' mean age was 69 years old and female as majority. Overall clinical evaluation scores show trends towards good functional outcome, while radiological scores show vary deviations of mechanical axis. Abnormal data distributions were found on WOMAC, OKS, CTFA and CTCA. 
Table 1. Subject characteristics of the study

\begin{tabular}{lc}
\hline Characteristic & Subjects (N=50) \\
\hline Age (in years) * & $69.68(6.65)$ \\
Gender & $12(24)$ \\
$\quad$ Male & $38(76)$ \\
$\quad$ Female & \\
Side & $30(60)$ \\
$\quad$ Right & $20(40)$ \\
$\quad$ Left & \\
Group & $25(50)$ \\
$\quad$ Computer-assisted & $25(50)$ \\
$\quad$ Conventional & $2(0-17)$ \\
Functional Scores & $46(35-48)$ \\
$\quad$ WOMAC* & \\
$\quad$ OKS* & $182(175-186)$ \\
Radiological Scores & $90.78(1.93)$ \\
$\quad$ CTFA* & $90(88-94)$ \\
$\quad$ CFCA* & \\
$\quad$ CTCA* & \\
\hline Proportional data is presented in: Frequency (percentage). \\
Numerical data is presented in: *Median (range); **Mean (SD). \\
WOMAC= Western Ontario Mac Master University Index; OKS= Oxford \\
Knee Score; CTFA= coronal tibiofemoral angle; CFCA= coronal femoral \\
component angle; CTCA= coronal tibial component angle. \\
\hline
\end{tabular}

Comparison of functional evaluation and radiological evaluation on each group is presented on Table 2. Based on statistical analysis, group of computer-assisted navigation TKA surgery was found to have significantly lower WOMAC score $(p<0.05)$ and higher OKS score $(p<0.05)$, if compared with conventional TKA surgery.

Table 2. Comparison of functional and radiological evaluation on both groups

\begin{tabular}{|c|c|c|c|}
\hline Clinical Evaluation & $\begin{array}{c}\text { Computer- } \\
\text { assisted }\end{array}$ & Conventional & p-value \\
\hline WOMACM & $1(0-12)$ & $3(0-17)$ & $0.040^{*}$ \\
\hline OKS ${ }^{\mathrm{M}}$ & $47(38-48)$ & $45(35-48)$ & $0.035 *$ \\
\hline \multicolumn{4}{|l|}{ Radiological } \\
\hline \multicolumn{4}{|l|}{ Evaluation } \\
\hline CTFA $^{\top}$ & $\begin{array}{c}2.08( \pm 1.49) \\
\text { varus }\end{array}$ & $\begin{array}{c}2.84( \pm 1.37) \\
\text { varus }\end{array}$ & 0.068 \\
\hline CFCA $^{\mathrm{M}}$ & $1(0-5)$ & $2(0-5)$ & 0.243 \\
\hline CTCA $^{\text {M }}$ & $1(0-2)$ & $1(0-4)$ & 0.467 \\
\hline
\end{tabular}

T)Data is presented in mean (SD), and analyzed with Independent T-test. M)Data is presented with median (range), and analyzed with MannWhitney test.

*significant value $\mathrm{p}<0.05$

WOMAC = Western Ontario Mac Master University Index; OKS= Oxford Knee Score; $C T F A=$ coronal tibiofemoral angle; $C F C A=$ coronal femoral component angle; $\mathrm{CTCA}=$ coronal tibial component angle.
On radiological evaluation, our study found no significant difference on the results of CTFA, CFCA and CTCA (Table 2). However, greater angle of deviation is observed in the conventional TKA surgery group in regards of CTFA mean and standard deviation comparison, and also CFCA median and range comparison (Figure 2).

Looking only at subjects with mechanical axis deviation of less than $\pm 3^{\circ}$ from neutral on both groups, there was no significant difference between computer-assisted and conventional group. Nevertheless, proportions with deviation of less than 3 o were found more in the group using computer navigation compared to conventional methods.

Table 3. Proportion comparison of subjects with mechanical axis deviation of less than $\pm 3^{\circ}$ on both groups

\begin{tabular}{lccc}
\hline Radiological Evaluation & $\begin{array}{c}\text { Computer- } \\
\text { assisted }\end{array}$ & $\begin{array}{c}\text { Conventi } \\
\text { onal }\end{array}$ & $\begin{array}{c}\text { p- } \\
\text { value }\end{array}$ \\
\hline CTFA $^{*}$ & $20(80 \%)$ & $17(68 \%)$ & 0.333 \\
CFCA $^{* *}$ & $23(92 \%)$ & $22(88 \%)$ & 1.000 \\
CTCA $^{* *}$ & $25(100 \%)$ & $24(96 \%)$ & 1.000 \\
\hline Data is presented in frequency (percentage) & & \\
${ }^{*}$ Analysis was done with Chi-square test & \\
${ }^{* *}$ Analysis was done with Fisher exact test & \\
CTFA= coronal tibiofemoral angle; CFCA= coronal femoral component \\
angle; CTCA= coronal tibial component angle.
\end{tabular}

\section{Discussion}

Population of our patients have the mean age of 69.68 years old with the majority of female gender. This is in accordance with epidemiology review of knee arthroplasty in Asia region by Singh. ${ }^{9}$ Our study showed good clinical evaluation in both functional score of patients who had computer-assisted TKA surgery. This good outcome was also observed in study by Hoffart et al. and Ishida et al. who evaluate 5-year clinical outcome by using Knee Society Knee Scoring System. ${ }^{10,11}$ On the contrary, some other studies shown no significant difference on clinical outcome in short to mid-term follow up. ${ }^{12-14}$ Although, better overall results were also observed on the computer-assisted group.

Turning to radiological outcome, study by Dutton et al. and Selvanayagam et al. found significant better coronal-plane mechanical 
alignment in patients underwent computerassisted navigation TKA surgery. ${ }^{8,15}$ However, our study found better scoring at all three parameters on computer-assisted navigation TKA surgery; although, this data is not statistically significant.

Similar finding with our study was also observed in study by Pacheco et al. ${ }^{16}$ In their study, the surgeon was already expert in conventional TKA, but had just been trained in computerassisted navigation TKA. This situation is also reflected in our study. The surgeon in our study is much more experienced in conventional TKA, despite already trained and accustomed with computer-assisted navigation surgery.

Additionally, our study only measures mechanical alignment from coronal plane. Study by Huffart et al. stated that other parameter measurement, such as mechanical alignment on sagittal plane, rotational alignment, and soft tissue balance are also important to determine the success rate of TKA surgery. ${ }^{10}$

Our data shows greater number of subjects within \pm 3 o deviations in the computer-assisted group compared to the conventional group. Similar results were also found in study by Dutton et al. ${ }^{8}$ However, their study found significant result in CTFA deviation and sagittal tibia component angle. This accentuate the importance of sagittalplane mechanical alignment measurement. Additionally, we did not measure preoperative radiographic value and may include patients with femoral bowing and tibial bowing in our study. As Lee et al. mentioned in their study, femoral bowing may lead to excess deviation after surgery. ${ }^{17}$

There are some limitations in our study that should be noted. As mentioned previously, we did not measure preoperative radiographic value, and therefore, patients with tibia and/or femoral bowing may be included in our study. The unavailability of preoperative data also hinder us from comparing it with post-operative data. Linguistic bias may exist due to the use of translated, but not standardized, self-assessed scoring system. Another limitation is the relatively short follow-up time (6 months), which preclude us from evaluating the longterm functional outcome.

The results of this study indicate the advantage of using a computer assisted navigation system in TKA operations, in terms of better functional outcome. These results illustrate that the quality of service for osteoarthritis patients can be further improved by involving the computer-assisted navigation system in TKA surgeries. The use of navigation systems in surgery is also still being developed and continuously updated to increase its effectiveness and efficiency. Therefore, this good technological progress needs to be adapted, understood and further investigated, especially in Indonesia. Authors hope that with further research and development of navigation systems, computerassisted surgery can become an option available to the wider community.

\section{Conclusions}

Patients who had computer-assisted navigation TKA procedure would benefit a better functional outcome compared to conventional TKA surgery, as measured with WOMAC and OKS. However, even though computer-assisted TKA showed better radiographic outcome, but there is no statistical difference on both methods in this study.

In order to obtain more objective result, double blind randomized control trial method can be recommended. Other functional scoring system, such as Knee Society Knee Score or Insall Knee Score may be also used. Additionally, other radiographic measurement such as preoperative radiographic measurement, mechanical alignment on sagittal plane, rotational alignment, and soft tissue balance should be included. The study can also be expanded by longer time of follow up, larger sample size, and observing the mortality rate, complication rate, as well as revision rate. 


\section{Ethical Clearance}

This study obtained ethical approval from the Saint Carolus Hospital Health Research Ethics Committee. All patients were consented to be included in the study and publication of the results. Research data used and analyzed in our study available from the corresponding author upon reasonable request.

\section{Conflicts of Interest}

The authors affirm no conflict of interest in this study

\section{Acknowledgment}

Authors would like to thank the Department of Orthopaedic and Traumatology, Faculty of Medicine, Universitas Indonesia and Department of Orthopaedic and Traumatology, Saint Carolus Hospital, Jakarta.

\section{References}

1. Ek ET, Dowsey MM, Tse LF, Riazi A, Love BR, Stoney JD, et al. Comparison of functional and radiological outcomes after computer-assisted versus conventional total knee arthroplasty: a matched-control retrospective study. J Orthop Surg (Hong Kong). 2008;16(2):192-6.

2. Pradhan NR, Gambhir A, Porter ML. Survivorship analysis of 3234 primary knee arthroplasties implanted over a 26-year period: a study of eight different implant designs. Knee. 2006;13(1):7-11.

3. Chauhan SK, Clark GW, Lloyd S, Scott RG, Breidahl W, Sikorski JM. Computer-assisted total knee replacement. A controlled cadaver study using a multi-parameter quantitative CT assessment of alignment (the Perth CT Protocol). J Bone Joint Surg Br. 2004;86(6):818-23.

4. Brin YS, Nikolaou VS, Joseph L, Zukor DJ, Antoniou J. Imageless computer assisted versus conventional total knee replacement. A Bayesian meta-analysis of 23 comparative studies. Int Orthop. 2011;35(3):331-9.
5. Decking R, Markmann Y, Fuchs J, Puhl W, Scharf HP. Leg axis after computer-navigated total knee arthroplasty: a prospective randomized trial comparing computer-navigated and manual implantation. J Arthroplasty. 2005;20(3):282-8.

6. Bauwens K, Matthes G, Wich M, Gebhard F, Hanson B, Ekkernkamp A, et al. Navigated total knee replacement. A meta-analysis. J Bone Joint Surg Am. 2007;89(2):261-9.

7. da Silva RR, Santos $A A$, de Sampaio Carvalho Junior J, Matos MA. Quality of life after total knee arthroplasty: systematic review. Rev Bras Ortop. 2014;49(5):520-7.

8. Dutton $A Q$, Yeo SJ, Yang KY, Lo NN, Chia KU, Chong HC. Computer-assisted minimally invasive total knee arthroplasty compared with standard total knee arthroplasty. A prospective, randomized study. J Bone Joint Surg Am. 2008;90(1):2-9.

9. Singh JA. Epidemiology of knee and hip arthroplasty: a systematic review. Open Orthop J. 2011;5:80-5.

10. Hoffart $\mathrm{HE}$, Langenstein $\mathrm{E}$, Vasak N. A prospective study comparing the functional outcome of computer-assisted and conventional total knee replacement. J Bone Joint Surg Br. 2012;94(2):194-9.

11. Ishida K, Matsumoto $T$, Tsumura N, Kubo S, Kitagawa A, Chin T, et al. Mid-term outcomes of computer-assisted total knee arthroplasty. Knee Surg Sports Traumatol Arthrosc. 2011;19(7):110712.

12. Huang TW, Lee $\mathrm{CY}$, Lin SJ, Peng $\mathrm{KT}$, Huang $\mathrm{KC}$, Lee MS, et al. Comparison of computernavigated and conventional total knee arthroplasty in patients with Ranawat type-II valgus deformity: medium-term clinical and radiological results. BMC Musculoskelet Disord. 2014;15:390.

13. Kamat YD, Aurakzai KM, Adhikari AR, Matthews D, Kalairajah Y, Field RE. Does computer navigation in total knee arthroplasty improve patient outcome at midterm follow-up? Int Orthop. 2009;33(6):1567-70.

14. Seon JK, Park SJ, Lee KB, Li G, Kozanek M, Song EK. Functional comparison of total knee 
arthroplasty performed with and without a navigation system. Int Orthop. 2009;33(4):987-90. 15. Selvanayagam R, Kumar V, Malhotra R, Srivastava DN, Digge VK. A prospective randomized study comparing navigation versus conventional total knee arthroplasty. J Orthop Surg (Hong Kong). 2019;27(2):2309499019848079.

16. Pacheco AP, Filho OT, da Mota EARF, Brambilla AC. Navigated Versus Mechanical Knee Replacement: Comparison between Initial Navigated and Conventional Cases Performed by the Same Surgeon. Rev Bras Ortop. 2012;47(3):359-62.

17. Lee $C Y$, Lin SJ, Kuo LT, Peng KT, Huang KC, Huang TW, et al. The benefits of computerassisted total knee arthroplasty on coronal alignment with marked femoral bowing in Asian patients. J Orthop Surg Res. 2014;9:122. 